\title{
Toxocara canis: una zoonosis frecuente a nivel mundial
}

\section{Toxocara canis: A worldwide frequent zoonosis}

Fecha recepción: 4 de julio de 2015

Fecha aceptación: 27 de octubre de 2015
Ana Carolina Rojas-Salamanca' María Camila León-Bustamante ${ }^{2}$ Olga Rocío Bustamante-Saavedra ${ }^{3}$

\section{Resumen}

La toxocariasis, causada principalmente por Toxocara canis, es una de las zoonosis más comunes a nivel mundial; se presenta con mayor frecuencia en niños, asociada a condiciones desfavorables de higiene, hacinamiento, convivencia con perros parasitados, el nivel socioeconómico, la ubicación geográfica y los entornos en los cuales los animales depositan sus heces, lo que se convierte en un gran foco de contaminación para los humanos. El Toxocara canis ingresa al ser humano por contacto directo con heces de perro o por contaminación de alimentos. La carga parasitaria es de vital importancia, ya que está relacionada directamente con la gravedad de la enfermedad, con los diferentes síndromes que se producen y con la respuesta inmune desencadenada por el organismo, teniendo en cuenta el ciclo de vida que se lleva a cabo en el organismo humano. Para esta enfermedad no se puede realizar un diagnóstico por técnicas coproparasitológicas, por lo cual es necesario utilizar otros métodos, como el aumento de leucocitos con presencia de eosinofilia, la prueba de ELISA y, en algunos casos, por medio de biopsias.

Palabras clave: contaminación (DeCS); larva migrans; Toxocara canis; zoonosis.

\begin{abstract}
The toxocariasis is one of the most common zoonoses worldwide mainly caused by Toxocara canis, occurs most often in children associated with unfavorable conditions of hygiene, overcrowding, living with dogs parasitized, low socioeconomic status, bad geographic location and within environment in which animals deposit their feces, becoming a major source of contamination for humans. These parasites enter in the human beings accidentally, by direct contact or food contamination with dog feces.

The parasite load is of vital importance as it is directly related to the severity of the disease, with different syndromes that occur and with the immune response discharged by the body, given the life cycle wich

1 Esp. Universidad de Boyacá (Tunja-Colombia).

2 Esp. Universidad de Boyacá (Tunja-Colombia).

3 M. Sc. Universidad Pedagógica y Tecnológica de Colombia (Tunja-Colombia).olga.bustamante@uptc.edu.co.
\end{abstract}


takes place within the human organism. This disease cannot be diagnosed by coproparasitological techniques, by which is required other methods such as the leukocyte with eosinophilia increase detection, also the ELISA proof and in some cases by biopsys.

Keywords: contamination (DeCS); larva migrans; Toxocara canis; zoonoses. 


\section{Introducción}

A lo largo de su historia, la humanidad ha venido estableciendo un estrecho contacto con los animales, lo que ha generado un aumento considerable en el desarrollo de diferentes enfermedades parasitarias y zoonóticas; la OMS ha notificado alrededor de 200 zoonosis, de las que, aproximadamente, 50 son trasmitidas al ser humano por caninos, siendo una de las más frecuentes a nivel mundial la infección producida por Toxocara canis (1).

Toxocara canis es un nemátodo que habita en el intestino delgado del perro, que es el huésped definitivo (2); sus huevos pueden sobrevivir aproximadamente 3 años en condiciones ambientales favorables para ellos, por lo cual se pueden encontrar en el suelo de diferentes zonas habitadas por el hombre; es por esto que el suelo es considerado la principal fuente de infección para los humanos, sobretodo en niños que tienen hábitos de geofagia, que juegan en los parques y que conviven con perros que están parasitados. De esta manera, la forma adulta del parásito ingresa en el hombre y se aloja en su intestino, produciendo síndromes de larva migrans, ya sea visceral, neurológico, ocular y encubierta (3).

Según datos establecidos por la OMS, la toxocariasis se encuentra ampliamente distribuida a nivel mundial, siendo endémica en la mayor parte de América, África y Asia, donde afecta principalmente a personas de estratos socioeconómicos bajos, debido a las condiciones de higiene desfavorables (1), al hacinamiento, a la convivencia con perros enfermos, a la ubicación de las residencias y a los entornos en los cuales los animales depositan sus heces, lo que se convierte en un gran foco de contaminación para los humanos, ya sea por contacto directo o por consumo de alimentos previamente contaminados con heces (4); además, donde no se realizan campañas de educación sanitaria en las que se expliquen los diferentes factores de riesgo y cómo prevenirlos; campañas de educación que deberían ser promovidas a nivel mundial en diferentes comunidades para contribuir a la disminución de la enfermedad, como se ha hecho con muchas otras enfermedades endémicas.
Es importante tener en cuenta que esta patología no se puede diagnosticar mediante técnicas coproparasitológicas, ya que las larvas, una vez invaden al ser humano, no llegan a su estado adulto y no producen huevos; por ello el diagnóstico de esta enfermedad se realiza principalmente por la medición de anticuerpos antitoxocara, por medio de la técnica de ELISA, por la aparición de eosinofilia producida por la presencia del parásito y por biopsias del tejido infectado (4).

Teniendo en cuenta los diferentes mecanismos por los cuales el ser humano puede adquirir la infección producida por este parásito, las condiciones que este necesita para desarrollarse y su alta prevalencia a nivel mundial, surge gran inquietud por saber cuáles son las consecuencias de la transmisión zoonótica de este parásito en la salud de los seres humanos.

\section{Marco teórico}

Toxocara canis es un nemátodo que habita en el intestino delgado de los caninos, y es endémico en todo el mundo; puede producir infección en el ser humano a partir de la ingestión de los huevos presentes en la tierra, verduras crudas y alimentos contaminados con las heces de perro (3). Este parásito pertenece al grupo de los enteroparásitos acáridos capaces de infectar al hombre accidentalmente, por lo cual la infección que produce es considerada una zoonosis; en este grupo se incluyen parásitos como Toxocara cati, Toxocara vitolorum, Toxocara canis y demás parásitos del género Toxocara que han sido reportados como zoonóticos en el ser humano (5).

Toxocara canis pertenece al phylum nematoda; es un nematodo parásito de cuerpo cilíndrico y no segmentado que mide entre 5 y $15 \mathrm{~cm}$. de longitud; es frecuente y casi universal del intestino delgado de caninos, zorros y lobos (5). La hembra de Toxocara canis pone alrededor de 200.000 huevos al día, solo en el intestino delgado del perro, que es el único huésped definitivo; tanto machos como hembras, desde los 20 días de nacidos hasta 1 año de edad, dispersan huevos de Toxocara canis; las hembras mayores de 1 año durante el celo, la pre- 
ñez o la lactancia, diseminan los huevos del parásito (5).

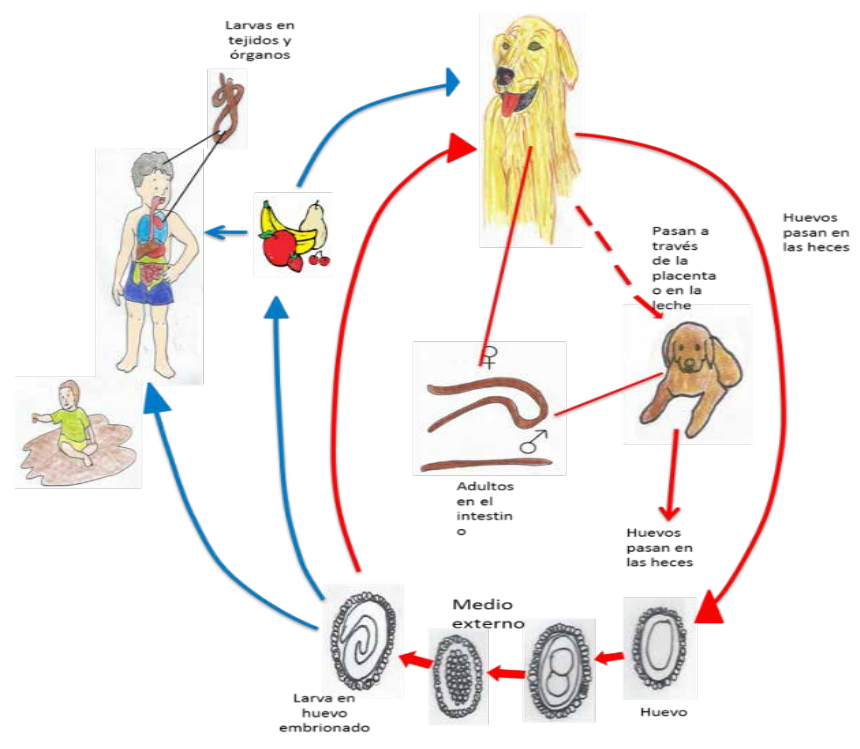

Figura 1. Ciclo de vida de Toxocara canis.

Para Toxocara canis, el suelo es un gran reservorio, donde los huevos evolucionan hasta el estadio juvenil (L2), lo que les permite permanecer estables durante uno a tres años; esto eleva la posibilidad de que el ser humano se infecte. La ingestión en caninos es por vía oral, el consumo de huevos infectados es la causa más común, pero se puede deber también por la ingesta de hospedadores infectados con el parásito. Los huevos eclosionan en el intestino delgado proximal; las larvas en estadio dos atraviesan la mucosa intestinal, hasta llegar a la circulación portal, donde Ilegan al hígado, y siguiendo la circulación llegan al corazón, pulmón y tráquea; de la tráquea son nuevamente ingeridos, y en el intestino mudan hasta alcanzar la madurez sexual, donde se producen los huevos no embrionados; estos huevos son eliminados a través de las heces y se desarrollan en el suelo, el cual les brinda las condiciones necesarias para su desarrollo y crecimiento; según la literatura, el período de prepatencia se calcula en 30 días, desde la ingestión hasta la eliminación $(5,6)$,

La infección en el humano se da cuando este ingiere los huevos embrionados; estos ingresan al organismo, se convierten en larvas e invaden la pared intestinal, distribuyéndose por diferentes órganos del cuerpo humano, lo cual produce el síndrome de larva migrans, debido a que estos parásitos no pueden evolucionar hacia formas adultas en el ser humano y quedan en su forma larval (7).

En los perros adultos el ciclo de infección es igual hasta la migración al pulmón, pero en estos los huevos llegan a la circulación y de allí pasan a los órganos y los músculos, donde las larvas pueden sobrevivir durante varios años.

\section{Enfermedad}

Las zoonosis son todas aquellas enfermedades transmisibles de forma natural de los animales a los humanos y viceversa (1). La OMS ha establecido que la toxocariosis se encuentra distribuida a nivel mundial, siendo endémica en la mayor parte de los países de América, África y Asia; esta es una de las enfermedades más prevalentes a nivel mundial y está relacionada con diferentes factores de riesgo a los cuales las personas se encuentran expuestas diariamente, como lo son el nivel socioeconómico, la ubicación geográfica del país, las viviendas en las cuales hay mascotas parasitadas, condiciones bajas de higiene, hábitos de geofagia en los niños, principalmente, y condiciones de hacinamiento (4).

Una vez el parásito ingresa al ser humano, se desarrolla en larva y puede migrar a diferentes órganos, como los ojos, el hígado, los riñones y los pulmones, y así se produce el síndrome de larva migrans, que puede ser visceral, ocular, neurológico y encubierta, y otras patologías (5).

La infección producida por Toxocara canis en el ser humano se presenta en tres fases diferentes, y la gravedad de la enfermedad depende del número de parásitos, del sitio de la infección y de la respuesta inmune del huésped. La fase aguda se produce cuando en el estómago de la persona que ha ingerido accidentalmente el huevo del parásito se liberan las larvas, con ayuda de los jugos gástricos y de las enzimas pancreáticas, larvas que por circulación sanguínea y linfática migran a diferentes órganos. Una vez terminada la fase aguda se presenta la fase latente, en la cual el parásito es atacado por el sistema inmunológico del ser humano y es 
Ilevado a un estado de latencia en el músculo, con lo cual no se van a producir ni signos ni síntomas característicos de la enfermedad; entre mayor sea la cantidad de parásito ingerido, va a ser mayor la respuesta inmune producida por el organismo, lo cual va a desencadenar una respuesta inflamatoria y, como consecuencia, esta fase de latencia lleva al paciente al desarrollo de una fase crónica de la enfermedad. En la fase crónica, por la respuesta inflamatoria desencadenada, el parásito se ubica en diferentes órganos y tejidos del hospedero accidental; como consecuencia de esto se van a producir los diferentes síndromes de larva migrans (7).

El síndrome de larva migrans visceral suele ser autolimitado; se presenta con mayor frecuencia en niños y cursa con manifestaciones clínicas, como lo son la hepatitis y la enfermedad pulmonar, presentando síntomas como hepatomegalia, tos, dificultad respiratoria y asma; en el corazón se puede producir miocarditis, y en algunos casos insuficiencia cardiaca; en la piel se evidencia una dermatitis atópica, y cuando se presenta de forma entérica se caracteriza por anorexia, náuseas, vómito, fiebre y dolor abdominal (5). El síndrome de larva migrans ocular es una de las formas clínicas más graves de la enfermedad; es común en niños mayores de 10 años, y también se puede presentar en adultos con menor frecuencia; desencadena síntomas como el estrabismo y la pérdida parcial o total de la visión $(8,9)$.

La neurotoxocariosis es más común en menores de 5 años y se da como consecuencia de la migración larval del parásito hacia el cerebro, donde se producen lesiones necróticas que tienden a confundirse con pequeños tumores cerebrales; además, por los síntomas presentados, como las convulsiones, suele ser confundida con trastornos epilépticos y meningitis; esta similitud con otras patologías hace más complejo diagnosticar la neurotoxocariosis (5, $10)$.

En el síndrome de larva migrans encubierta, el parásito se ubica en el músculo estriado sin causar signos y síntomas característicos de la infección, o puede ocurrir que una persona que haya tenido una primoinfección presente nuevamente los signos y síntomas de la enfermedad, debido a que las larvas pueden sobrevivir por meses, años o incluso de por vida en el organismo (7).

En diferentes países en los cuales han realizado estudios sobre esta enfermedad ha surgido la hipótesis de la relación de la toxocariasis con el desarrollo de asma, sobretodo en pacientes pediátricos; estudios realizados han demostrado que los pacientes que tienen contacto con animales parasitados por Toxocara canis sufren más episodios asmáticos al mes que los pacientes que no conviven con este factor de riesgo; pero en la mayoría de los estudios realizados no se ha podido demostrar la asociación de la infección por Toxocara canis y el desarrollo de asma, debido a que se ha realizado poca investigación sobre este tema, lo que no permite establecer una afirmación sobre esta hipótesis $(3,11,12)$.

Los síndromes producidos por este parásito son, generalmente, erradicados con antihelmínticos, pero el método más efectivo para reducir los altos índices de la enfermedad a nivel mundial es la profilaxis, que se basa en evitar la contaminación del suelo y los alimentos con heces de perros, control veterinario de las mascotas y disminuir los factores de riesgo tanto en humanos como en las mascotas (3).

\section{Diagnóstico}

Para el diagnóstico de Toxocara canis se debe tener en cuenta el tipo de toxocariasis que está cursando la persona infectada, conocer la historia clínica, identificar las características clínicas del paciente y su situación socioeconómica, para determinar los posibles factores predisponentes de la enfermedad y poder realizar un diagnóstico diferencial. Lo ideal en este tipo de infecciones es conocer e identificar la fuente principal de la infección, para reducir el riesgo de posibles infecciones futuras o una diseminación a la comunidad, así como también identificar y escoger el mejor método para tener un correcto y oportuno diagnóstico $(5,13)$.

En los animales que se encuentran parasitados se realiza el diagnóstico por el método coproparasitológico, ya que los huevos embrionados de Toxocara canis se pueden observar en el examen 
microscópico; esta es la técnica más común para realizar el diagnóstico en los animales parasitados (14). En el ser humano no es posible realizar un diagnóstico por técnicas coproparasitológicas, debido al ciclo de vida que lleva el parásito dentro del organismo humano, por lo cual es indispensable utilizar métodos alternativos.

Con el gran avance científico de los últimos años, se han creado nuevas técnicas para realizar el diagnóstico de diferentes enfermedades; teniendo en cuenta el tipo de toxocariosis se utilizan diferentes métodos para su diagnóstico, como la biopsia, pero como este es un procedimiento invasivo para el paciente, se ha optado por las técnicas inmunológicas (4). Actualmente se realiza el diagnóstico serológico de la toxocariasis por medio de la técnica inmunoenzimática ELISA, que va a detectar la presencia de antígenos secretados por las larvas de Toxocara canis, permitiendo diagnosticar las diferentes presentaciones clínicas de la enfermedad (15).

Hay que tener en cuenta algunos parámetros importantes, como la presencia de eosinofilia y la observación de las larvas enquistadas en diferentes órganos del cuerpo humano, que en la mayoría de los casos tiende a ser confundida con lesiones tumorales, por lo cual se debe realizar un manejo quirúrgico para corroborar el diagnóstico (5).

Es importante, para realizar un correcto diagnóstico de esta patología, observar detalladamente tanto los signos como los síntomas de la enfermedad, los cuales, en la mayoría de los casos, no son específicos y tienden a confundirse con otras patologías, por lo cual es de vital importancia tener un apoyo clínico de imagenología, para descartar un posible tumor, y de laboratorio clínico, para confirmar la enfermedad (5).

\section{Epidemiología}

Debido al incremento del número de casos de esta enfermedad, se han desarrollado diferentes estudios epidemiológicos que indican la presencia de la enfermedad a nivel mundial, como se relaciona en la siguiente tabla $(3,5,15,16)$.
Tabla I. Seroprevalencia de Toxocara canis a nivel mundial.

\begin{tabular}{|c|c|}
\hline Países & $\begin{array}{c}\text { Promedio de prevalencia } \\
\text { serológica de T. canis }\end{array}$ \\
\hline Grecia & $97.5 \%$ \\
\hline Alemania & $87.1 \%$ \\
\hline Bolivia & $83 \%$ \\
\hline Nepal & $81 \%$ \\
\hline Chile & $75 \%$ \\
\hline Indonesia & $68 \%$ \\
\hline Japón & $63.3 \%$ \\
\hline Irán & $63.3 \%$ \\
\hline Brasil & $60 \%$ \\
\hline Malasia & $54.5 \%$ \\
\hline Venezuela & $53 \%$ \\
\hline Colombia & $47.5 \%$ \\
\hline Cuba & $42 \%$ \\
\hline Argentina & $31 \%$ \\
\hline Turquía & $30.6 \%$ \\
\hline Egipto & $30 \%$ \\
\hline Perú & $22.46 \%$ \\
\hline USA & $20.6 \%$ \\
\hline México & $12.45 \%$ \\
\hline Londres & $6.3 \%$ \\
\hline Francia & $5 \%$ \\
\hline Suiza & $5 \%$ \\
\hline Corea & $5 \%$ \\
\hline España & $1.2 \%$ \\
\hline Caninos & $43 \%$ \\
\hline Ambiente & $77 \%$ \\
\hline
\end{tabular}

Fuente: Lozano D., Suárez E., Ortuño E., Cruz M., Córdova M., Jiménez G., Getaz L. Relación entre asma y toxocariasis en pacientes pediátricos en Cochabamba, Bolivia. Gaceta Médica Boliviana, 2011; 34(2): 76-79; Archelli S., Kozubsky L. Toxocara y Toxocariosis. Acta Bioquímica Clínica Latinoamericana, 2008; 42(3): 379-384; Ramírez C., Hernández A., Breña J., Yoshiyama C., Lu L., Alzamora B., Maguiña C. Pacientes con toxocariosis ocular atendidos en el Hospital Nacional Cayetano Heredia, el Hospital Nacional Arzobispo Loayza y el Instituto Nacional de Salud del Niño entre los 
años 1997 y 2010. Revista Acta Médica Peruana, 2010; 27(4): 250-256; Khazan H., Khazaei M., Seyyed S., Mehrabi A.. Prevalence of Toxocara spp. eggs in public parks in Teheran City, Iran. Iranian Journal of Parasitology, 2012; 7(3): 38-42.

Los altos grados de seropositividad para Toxocara canis, probablemente, estén relacionados con el alto porcentaje de viviendas con mascotas con condiciones de higiene dudosas, y en especial con calles y parques públicos que tienen condiciones sociales y medioambientales para una alta prevalencia de toxocariasis canina y su inminente riesgo al ser humano (3).

Podemos observar que esta zoonosis se encuentra ampliamente distribuida a nivel mundial, tanto en países desarrollados, subdesarrollados y en vía de desarrollo, lo cual se debe a la costumbre de tener perros de compañía que, generalmente, están contaminados o adquieren la enfermedad del ambiente, convirtiéndose en un gran foco de contaminación para el ser humano (7).

En países como Francia, Suiza, Corea y España se observa una baja prevalencia de la enfermedad, debido a que ejercen control de infecciones en caninos tanto domésticos como callejeros, lo cual reduce el porcentaje de contaminación hacia el ser humano, pero no erradica el riesgo, dado que el parásito se encuentra de forma natural en los suelos y en el ambiente $(16,17)$.

\section{Conclusiones}

En estudios recientes se relaciona la toxocariasis con la presencia del asma, pero esto no ha sido confirmado, por lo cual es recomendable realizar estudios en diferentes poblaciones para comprobar este postulado.

Los altos índices de toxocariasis a nivel mundial destacan la importancia de tomar medidas de prevención y control en los diferentes lugares que constituyen factor de riesgo para las personas.

Teniendo en cuenta la diversidad en cuanto a la presentación clínica de la toxocariasis, es indispensable ejecutar un oportuno y correcto diagnóstico, agotando todas las posibilidades para confirmar la enfermedad.

A pesar de que la toxocariasis se produce de forma accidental, es necesario tener en cuenta los diferentes factores de riesgo de contaminación para el ser humano, con el fin de obtener información para enfocar el diagnóstico de la enfermedad.

\section{Referencias}

(1) Gallardo J., Camacho S. Infección por Toxocara canis y factores de riesgo en niños de la comunidad Agua Azul, Estado Yaracuy. Revista de Enfermería y otras Ciencias de la Salud, 2012; 5(1): 21-27.

(2) Reyna J., Limón A., Nova R. Invasión intestinal por Toxocara canis como diagnóstico diferencial de linfoma: informe de un caso. Revista de Enfermedades Infecciosas y Microbiología, 2007; 27(3): 100-102.

(3) Lozano D., Suárez E., Ortuño E., Cruz M., Córdova M., Jiménez G., Getaz L. Relación entre asma y toxocariasis en pacientes pediátricos en Cochabamba, Bolivia. Gaceta Médica Boliviana, $2011 ; 34(2)$ : 76-79.

(4) Nieves A., Ortega B., Martínez M., Castejón O., Lares M., Ferret E. Estandarización de la técnica de ELISA para el diagnóstico inmunológico de toxocariasis humana. Boletín de Malariología y Salud Ambiental, 2012; 52(1): 21-32.

(5) Archelli S., Kozubsky L. Toxocara y Toxocariosis. Acta Bioquímica Clínica Latinoamericana, 2008; 42(3): 379-384.

(6) Martin U., Machuca P., Demonte M., Contini L. Estudio en niños con diagnóstico presuntivo de toxocariasis en Santa Fe, Argentina. Revista Médica de Buenos Aires, 2008; 68(5): 353357.

(7) Roldán W., Espinoza Y., Huapaya P., Jiménez $S$. Diagnóstico de la toxocariasis humana. Revista Peruana de Medicina Ex- 
perimental y Salud Pública 2010; 27(4): 613620. DOI: http://dx.doi.org/10.1590/S172646342010000400019.

(8) Pivetti P. Ocular toxocariasis. International Journal of Medical Sciences, 2009; 6(3): 129-130. DOI: http://dx.doi.org/10.7150/ ijms.6.129.

(9) Cortez R., Ramírez G., Collet L., Giuliari G. Ocular parasitic diseases: a review on toxocariasis and diffuse unilateral subacute neuroretinitis. Journal of Pediatric Ophthalmology and Strabismus, 2010; 20(10): 1-10.

(10) Quattrocchi G., Nicoletti A., Marin B., Bruno E., Druet M., Preux P. Toxocariasis and epilepsy: systematic review and Meta-analysis. Public Library of Science, 2012; 6(8): 110. DOI: http://dx.doi.org/10.1371/journal. pntd.0001775.

(11) Getaz L., Samalvides F., Breña J., Torrejón D., Maguiña C. Relación entre toxocariosis y asma: estudio prospectivo en niños del hospital nacional Cayetano Heredia, Lima, Perú. Revista Acta Médica Peruana, 2007; 24(2): 8190.

(12) López M., Bojanich M., Jacobacci J., Sercic C., Michelini A., Alonso J. Toxocara canis y asma bronquial. Revista Médica de Buenos Aires, 2010; 70(1): 75-78.

(13) Rivarola M., Vuyk I., Riveros M., Canese A., Mico G. Toxocara canis en población pediátrica rural. Revista Pediátrica de la Asunción, 2009; 36(2): 122-126.

(14) Castillo J., Morales A., Molina E., Cepero O., Gutiérrez D., Fernández J. Prevalencia y factores que favorecen la presentación de Toxocara canis y ancylostoma caninum en canes de compañía. Revista Electrónica de Veterinaria, 2012; 13(6): 1-15.

(15) Ramírez C., Hernández A., Breña J., Yoshiyama C., Lu L., Alzamora B., Maguiña C. Pacientes con toxocariosis ocular atendidos en el Hospital Nacional Cayetano Heredia, el Hospital Nacional Arzobispo Loayza y el Instituto Nacional de Salud del Niño entre los años 1997 y 2010. Revista Acta Médica Peruana, 2010; 27(4): 250-256.

(16) Khazan H., Khazaei M., Seyyed S., Mehrabi A. Prevalence of Toxocara spp. eggs in public parks in Teheran City, Iran. Iranian Journal of Parasitology, 2012; 7(3): 38-42.

(17) Sariego I., Kanobana K., Rojas L., Speybroeck N., Polman K., Nuñez F. Toxocariasis in Cuba: a literature review. Public Library of Science, 2012; 6(2): 1-7. DOI: http://dx.doi. org/10.1371/journal.pntd.0001382.

(18) Romero C., García A., Mendoza G., Torres N., Ramírez N. Contaminación por Toxocara spp. en parques de Tulyehualco, México. Revista Científica FCV, 2009; 19(3): 253-256.

(19) Tanveer S., Yattoo G., Sofi B., Wani S., Dar P., Fomda B. Seroprevalence of Toxocariasis in children in Kashmir, India. Irianian Journal of Parasitology, 2008; 3(4): 45-50.

(20) Rateb A. Prevalence of Toxocara canis in dogs, North West bank of Palestine. Korean Journal of Parasitology, 2011; 49(2): 181 - 182. DOI: http://dx.doi.org/10.3347/kjp.2011.49.2.181.

(21) Choi D., Lim J., Choi D., Soo K., Woon S., Kim S., Choi Y., Huh S. Transmission of Toxocara canis via ingestion of raw cow liver: a cross sectional study in healthy adults. Korean Journal of Parasitology, 2012; 50(1): 23-27. DOI: http://dx.doi.org/10.3347/kjp.2012.50.1.23.

(22) Cihangir A. Visceral larva migrans among children in Kütahya (Turkey) an evaluation of playgrounds for t. canis eggs. The Turkish Journal of Pediatrics, 2010; 52(3): 158-162.

(23) De la Fe P., Dumenigo B., Brito A., Aguirar J. Toxocara canis y syndrome de larva migrans visceralis. Revista Electrónica de Veterinaria REDVET, 2006; 7(4): 1-42. 
(24) The center for food security and public health. Toxocariasis, larva migrans visceral, larva migrans ocular, granulomatosis parasitaria, retinitis ocular. College of Veterinary Medicine Journal, 2005; 5(3): 1-7.

(25) Despommier D. Toxocariasis: clinical aspects, espidemiology, medical ecology and molecular aspects. Clinical Microbiology Journal, 2003; 16(2): 265-272. DOI: http://dx.doi. org/10.1128/CMR.16.2.265-272.2003.

(26) Luzna A. Toxocariosis in children living in highly contaminated área. An epidemiological and clinical study. Acta Parasitology, 2005; 45(5): 40-42.

(27) Wisniewska L., Wozniakowska T., Sobolewska D., Markiewicz J., Wieczorek M. Analysis of the course and treatment of toxocariasis in chikdren-a long-term observation. Parasitology Research, 2012; 110(6): 2363-2371. DOI: http://dx.doi.org/10.1007/s00436-0112772-y.
(28) Elanie A., Carvalho A., Regina L., Rocha. Toxocariasis: visceral larva migrans in children. Jornal de Pediatria, 2011; 87(2): 100-110.

(29) González A., Gude F., Campos J., Garea M., Romero P., Rey J., et al. Toxocara infection seroprevalence and its relationship with atopic features in a general adult population. Int Arch Allergy Inmunology, 2006; 139(6): 317-324. DOI: http://dx.doi.org/10.1159/000091603.

(30) Coelho L., Silva M., Dini C., Giacon N., Novo N., Silveria E. Human toxocariasis: a seroepidemiological survey in schoolchildren of Sorocaba, Brazil. Mem Inst Oswaldo Cruz, 2004; 99(8): 533-537. DOI: http://dx.doi. org/10.1590/S0074-02762004000600002.

(31) López M., Martín G., Chamorro M., Alonso M. Toxocariasis in children form a subtropical región. Revista Médica de Buenos Aires, 2005; 65(4): 226-230. 